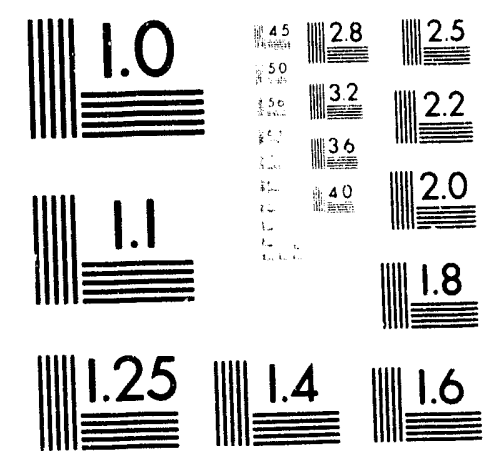



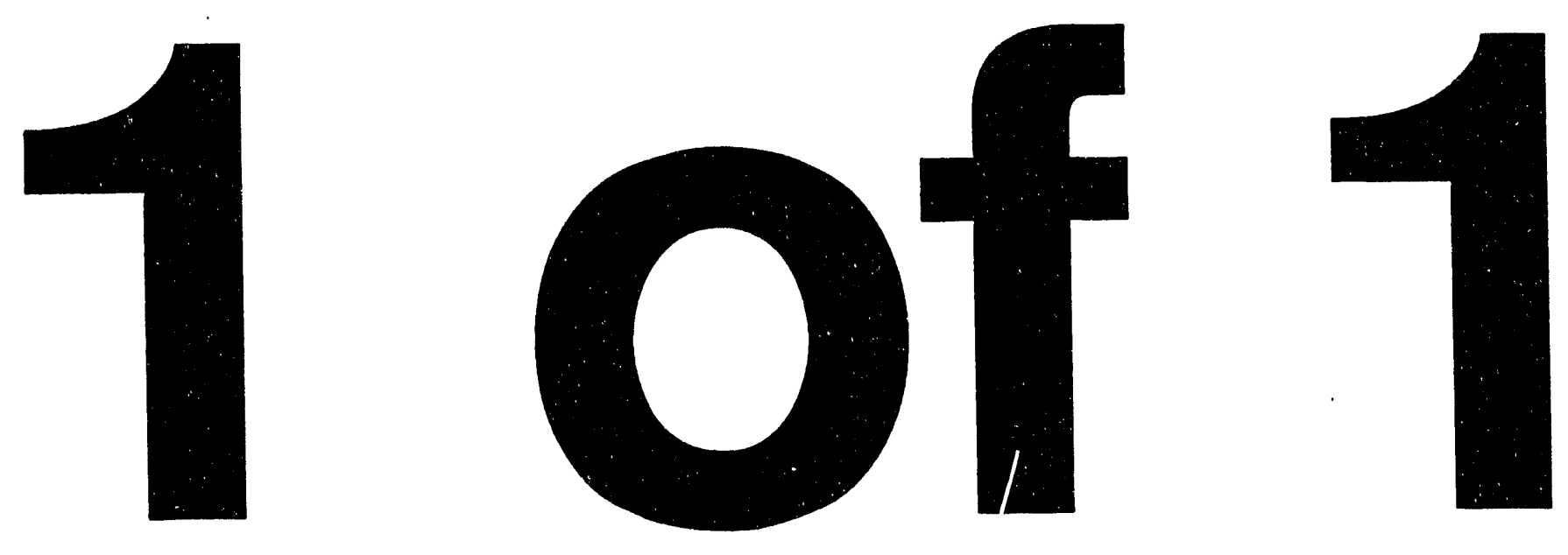
UCRL-JC-113091

PREPRINT

\title{
Neutron Detector for Fusion Reaction-Rate Measurements
}

\author{
R. A. Lerche, D. W. Phillion, and G. L. Tietbohl
}

This paper was prepared for submittal to the SPIE Conference on Ultrahigh- and High-Speed Photography, Videography, and Photonics '93 July 11-16, 1993, San Diego, CA

September 3, 1993

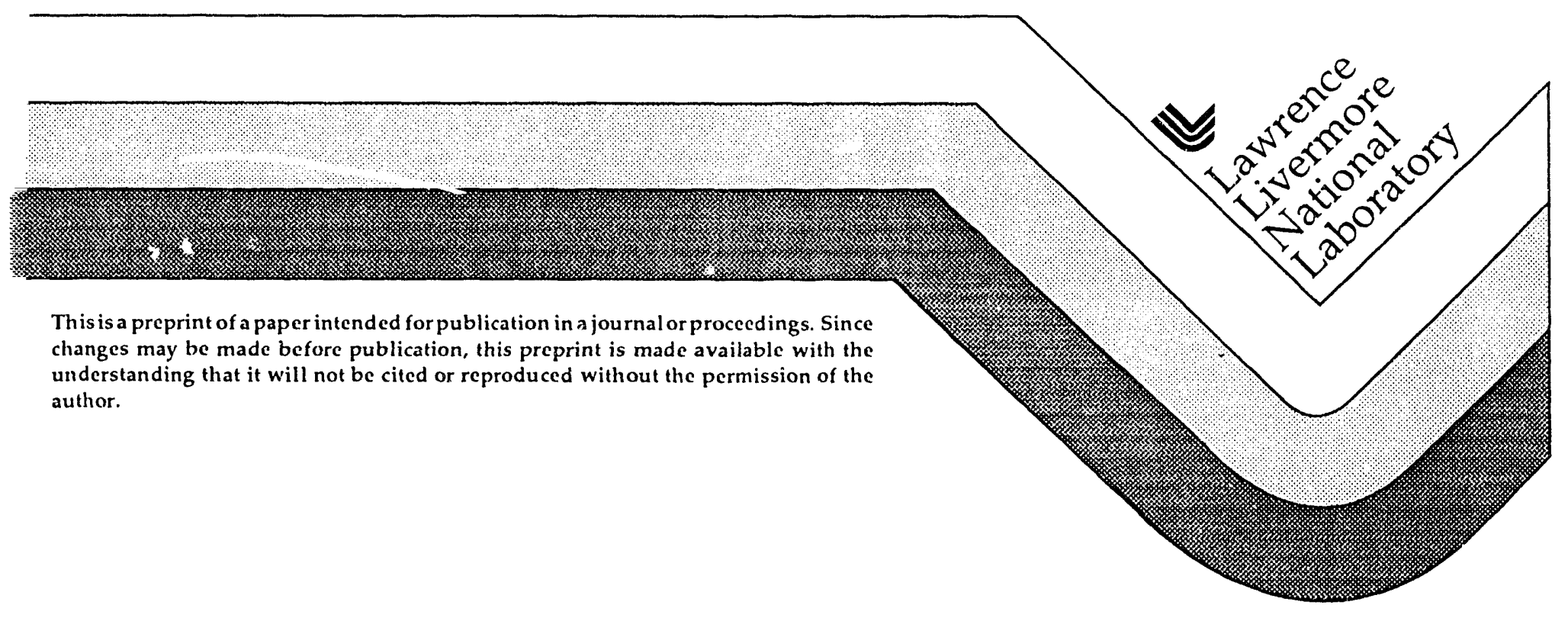

This is a preprint of a paper intended for publication in a journal or procecdings. Since changes may be made before publication, this preprint is made available with the understanding that it will not be cited or reproduced without the permission of the author. 


\section{I)IS( I.AIIIKK}

This document was prepared as an account of nork sponsored by an agency of the United States Ciovernment. Veither the I nited States (iovernment nor the I niversity of (alifornia nor any of their cmployees, makes any warranty, express or implied, or assumes any legal liability or responsibility for the accuracy, completeness, or usefulness of ans information, apparatus, product, or process disclosed, or represents that is use would not infringe privalcly ouned rights. Reference herein to any specific commercial products, process, or service by trade name, trademark. manufacturer, or wherwise, does not necessarily constitute or imply its endorsement, recommendation, or favoring by the I nited States Covernment or the I nivervity of Catifornia. The viens and opinions of authors expressed herein do not necessarily state or reflect those of the I nited states ciovernment thereof, and ha!l not be used for advertising or product endorsement purpeses. 


\title{
Neutron detector for fusion reaction-rate measurements
}

\author{
R. A. Lerche, D. W. Phillion, and (. L. Tietbohl \\ Lawrence Livermore National Laboratory \\ P. O. Box 5508, L-473, Livermore, CA 94550
}

\begin{abstract}
We have developed a fast, sensitive neutron detecior for recording the fusion reaction-rate history of inertial-confinement fusion (ICF) experiments. The detector is based on the fast rise-time of a commercial plastic scintillator (BC-422) and has a response $<25$ ps FWHM. A thin piece of scintillator material acts as a neutron-to-light converter. A zoom lens images light from the scintillator surface to a high-speed ( $15 \mathrm{ps)} \mathrm{optical} \mathrm{streak} \mathrm{camera} \mathrm{for} \mathrm{recording.} \mathrm{The} \mathrm{zoom} \mathrm{lens} \mathrm{allows} \mathrm{the} \mathrm{scintillator} \mathrm{to}$ be positioned between 1 and $50 \mathrm{~cm}$ from a target. The camera simultaneously records an optical fiducial pulse which allows the camera time base to be calibrated relative to the incident laser power. Bursts of x rays formed by focusing 20-ps, 2.5-TW laser pulses onto gold disk targets demonstrate the detector resolution to be $<25 \mathrm{ps}$. We have recorded burn histories for deuterium/ tritium-filled targets producing as few as $3 \times 10^{7}$ neutrons.
\end{abstract}

\section{INTRODUCTION}

This paper describes an application for a high-speed streak camera in inertial-confinement fusion (ICF) research. In ICF experiments, spherical targets filled with deuterium (DD) or a deuterium-tritium (DT) mixture are heated and compressed to conditions under which thermonuclear fusion occurs. At Livermore, we irradinte submillimeter targets with up to 30-kJ of energy at a wavelength of $0.351 \mu \mathrm{m}$ from the 10-beam Nova laser.' Our experimental goals are to achieve 100()$\times$ liquid density and temperatures of $10 \mathrm{keV}$ for confinement times on the order of $100 \mathrm{ps}^{2}$ During the plasma confinement time, fuel atoms undergo fusion, releasing energetic charged particles, neutrons, and photons. We are interested in measuring the fusion rate as a function of time relative to the incident drive radiation. This burn history depends on the coupling of he laser energy to the target, the hydrodynamics of the target implosion, and the plasma conditions during peak compression. Burn history is a sensitive indicator of our ability to accurately model energy transport between laser and target.

The reactions of interest in ICF experiments are

$$
\begin{aligned}
& \mathrm{D}+\mathrm{D} \rightarrow{ }^{3} \mathrm{He}(0.82 \mathrm{MeV})+\mathrm{n}(2.45 \mathrm{MeV}) \\
& \mathrm{D}+\mathrm{D} \rightarrow \mathrm{T}(1.01 \mathrm{MeV})+\mathrm{p}(3.02 \mathrm{MeV}) \\
& \mathrm{I}+\mathrm{T} \rightarrow{ }^{4} \mathrm{He}(3.56 \mathrm{MeV})+\mathrm{n}(14.03 \mathrm{MeV})
\end{aligned}
$$

where the particle energy is indicated in parentheses. Thermal motion of the reacting plasma ions causes a small energy spread in the otherwise monoenergetic spectra for the fusion products of these reactions. The charged particles are slowed by coulomb interactions with plasma ions and electrons before leaving the fuel. Most neutrons, however, escape the target without collision. Thus, the neutron temporal distribution at a point or:tside the target preserves burn history information with a time-of-flight cielay. For this reason, burn history may be measured with a fast neutron detector some distance outside the target. Since ronfinement times are on the rder of $100 \mathrm{ps}$, a de'ector with time resolution on the order of $20 \mathrm{ps}$ is highly desirable. given by

Thermal motion of the reacting plasma ions causes a ioppler broadening of the neutron energy spectrum, ${ }^{3}$ which is

$$
\left.\Delta E=\left\{\begin{array}{c}
82.5 \\
177
\end{array}\right\} \sqrt{T} \mid \begin{array}{l}
\mathrm{DD} \\
\mathrm{DT}
\end{array}\right\},
$$


where $T$ is the ion temperature (keV) and $\Delta E$ is the FWHM of the energy distribution (keV). For neutrons leaving a target at the same instant in time, the energy distribution causes a spread in their arrival times at a detector. The FWHM of this temporal spread $\Delta t_{\mathrm{T}}$ is given by

$$
\Delta t \mathrm{~T}=\left\{\begin{array}{l}
778 \\
122
\end{array}\right\} \frac{\mathrm{ps}}{\mathrm{m} \mathrm{keV}^{1 / 2}} \sqrt{T} \times d\left|\begin{array}{c}
\mathrm{DD} \\
\mathrm{DT}
\end{array}\right|
$$

where $d$ is the distance between the target and neutron detector. To keep this time spread small, the detector must be placed close to the target. For example, to keep it below 20 ps requires

$$
\begin{aligned}
& d \leq\left\{\begin{array}{rr}
2.6 \mathrm{~cm} & \text { DD } \\
16.4 \mathrm{~cm} & \text { DT }
\end{array}\right\} \text { for } T=1 \mathrm{keV} \\
& d \leq\left\{\begin{array}{ll}
0.8 \mathrm{~cm} & \text { DD } \\
5.2 \mathrm{~cm} & \text { DT }
\end{array}\right\} \text { for } T=10 \mathrm{keV} .
\end{aligned}
$$

The penetrating nature of the neutrons allows substantial shielding against target debris and $x$ rays without significant loss of neutron signal. This makes short target-to-detector distances practical.

The relatively slow speed of the neutrons requires a thin detector to maintain temporal resolution. In a thick detector, uncertainty in the neutron interaction point results in an uncertainty in the instant of the interaction. Thus, the time a neutron takes to pass through a detector limits the detector resolution. Again, for a 20-ps resolution, the thickness $l$ of the detector must be less than

$$
l \leq\left\{\begin{array}{ll}
0.4 \mathrm{~mm} & \mathrm{DD} \\
1.0 \mathrm{~mm} & \mathrm{DT}
\end{array}\right\}
$$

The less stringent distance and thickness requirements for the 14-MeV DT neutrons are a distinct advantage for using DTfilled capsules to make burn history measurements. Furthermore, the larger fusion cross section for DT neutrons results in a DT-neutron yield nearly 100 times greater than for a hydrodynamic equivalent DD-filled target, and thereby reduces statistical uncertainty in measurements.

Often, a "bang time" and "burn width" are reported instead of a full burn history because of limited detector temporal resolution. Detectors with a relatively poor $1-n s$ response can determine average neutron emission time (bang time) with an accuracy of better than $\pm 100 \mathrm{ps} .{ }^{4}$ Since these detectors typically have larger volumes, they also have greater sensitivity. Faster 100- 10 200-ps detectors have marginal resolution for making detailed burn history measuremeits. But they can often provide an estimate for the burn duration of the fuel. ${ }^{5.6}$ These detectors tend to be smaller and less sensitive.

In this article, we describe a streak camera based neutron detector. It was specifically developed to be a sensitive, highresolution detector for measuring the burn history of ICF targets. Its response is $\sim 25 \mathrm{ps} \mathrm{FWHM,} \mathrm{and} \mathrm{it} \mathrm{is} \mathrm{sensitive} \mathrm{enough} \mathrm{to}$ record a statistically meaningful burn history for targets yielding only $3 \times 10^{7}$ DT neutrons.

\section{DETECTOR}

\subsection{Concept}

Our detector is based on the fast rise time of a plastic scintillator. As monoenergetic neutrons expand radially outward from a small $\left(\sim 100-\mu \mathrm{m}\right.$ diameter) ICF target, ${ }^{7}$ they preserve reaction-rate information with a time-of-flight delay. A thin piece of $\mathrm{BC}-422^{8}$ plastic scintillator converts neutron kinetic energy to light. As the neutrons pass through the scintillator, some of them have elastic collisions with hydrogen nuclei. The recoil protons quickly transfer their kinetic energy to luminescent states in the scintillator. For BC. 422 the light output has a rise time $<20$ ps and a decay time of $\sim 1.2$ ns. The temporal distribution of the emitted light is the convolution of the neutron temporal istribution with the scintillator response. Burn history information is encoded in the leading edge of the output pulse. 


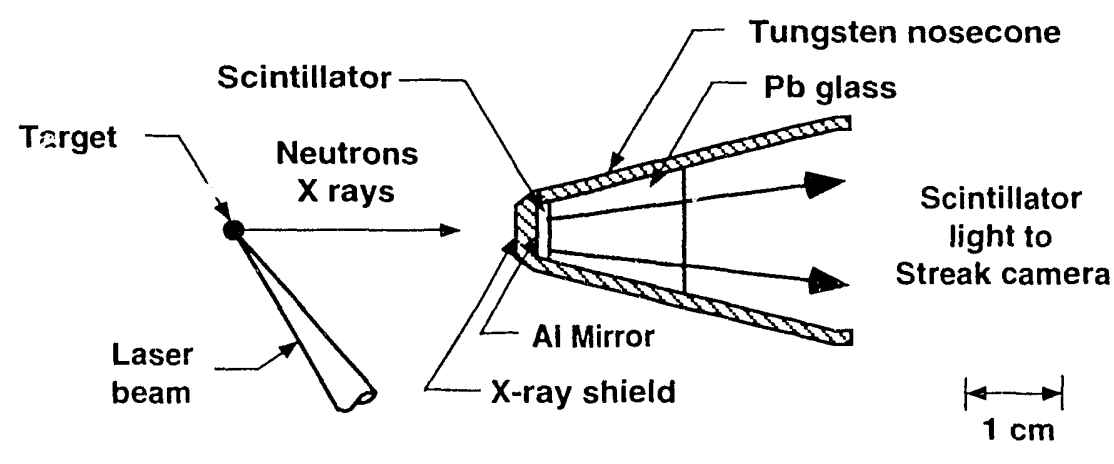

Figure 1. Scintillator configuration. Scintillator must be thin and close to the target to control temporal dispersion.

\subsection{Design Considerations}

Design goals for our detector system are speed, sensitivity, and ease of use. Detector speed is to approach $20 \mathrm{ps}$, the speed of the scintillator rise time. This requires using a thin scintillator close to the target; it also requires that the scintillator light be coupled to a fast readout device with a low dispersion technique. To be useful for Nova experiments, the detector must be sensitive enough to record the burn history for targets producing as few as $10^{\prime \prime}$ DT neutrons. Because there is limited access to the inside of the Nova target chamber, routine detector operation must not require chamber access.

To meet the speed requirement, we chose an optical streak camera as the recording device. The camera which is based on the RCA C73435 image tube is operated with a wide $(1-\mathrm{mm})$ input slit to enhance system sensitivity. Careful focusing of the electron optics of the tube allows us to achieve 1()- to 15-ps camera response even with this relatively wide slit." ('seful scintillator surface area is limited by the available photocathode area $(1 \mathrm{~mm} \times 2.5 \mathrm{~mm})$.

\subsection{Detector Description}

Figure 1 shows the experimental arrangement for the scintillator. A $6-\mathrm{mm}$ diameter, $1.5-\mathrm{mm}$ thick piece of $\mathrm{BC}-422 \mathrm{is}$ housed in a Hevimet (90\% tungsten) nosecone. The front of the nosecone, which is 3-mm thick, shields the scintillator from target $x$ rays, scattered laser light and target debris. An aluminized mirror deposited on one surface of the scintillator doubles the light output directed towards the streak camera. A piece of $\mathrm{Pb}$ glass shields the back side of the scintillator from scattered $x$ rays. This nosecone design allows the scintillator to be positioned within $1 \mathrm{~cm}$ of a target.

The detector system is shown in figure 2 . The scintillator, which emits light with wavelengths between 350 and $450 \mathrm{~nm}$, is mounted in a retractable nosecone. The scintillator can be positioned at any point between 1 and $50 \mathrm{~cm}$ from target chamber center. For storage, the nosecone can be retracted to a point $80 \mathrm{~cm}$ from target chamber center. An achromatic $\mathrm{f} / 2 \mathrm{zoom}$ lens relays the scintillator image along a 4-m optical path to the S-20 photocathode of the streak camera. This arrangement allows the camera to be totally outside the chamber. Lens coupling was chosen to give minimal temporal dispersion. In contrast, a 4-m coupler made of graded-index optical fibers would produce about $80 \mathrm{ps}$ of dispersion. Baffling and a light shield prevent scattered laser light from entering the lens system. Scintillator light passes through a glass window at the vacuum chamber wall. Components inside the chamber can operate at eithe." vacuum or atmospheric pressure. The streak camera image is recorded with a C.CD camera." The relatively long distance between target and streak camera is an advantage of this configuration. Background caused by target $\mathrm{x}$ rays and neutrons interacting in the streak camera and the CCD readout decreases inversely with the square of the distance.

Besides the neutron signal, the streak camera also records an optical fiducial pulse. This allows the time base of the camera to be related to the time of the incident Nova laser beams. The Nova laser facility generates a 100-ps, $0.53-\mu \mathrm{m}, 0 \mathrm{ptical}$ fiducial pulse that is synchronized with the main laser pulse. The fiducial pulse is split and fanned out to various diagnostic 


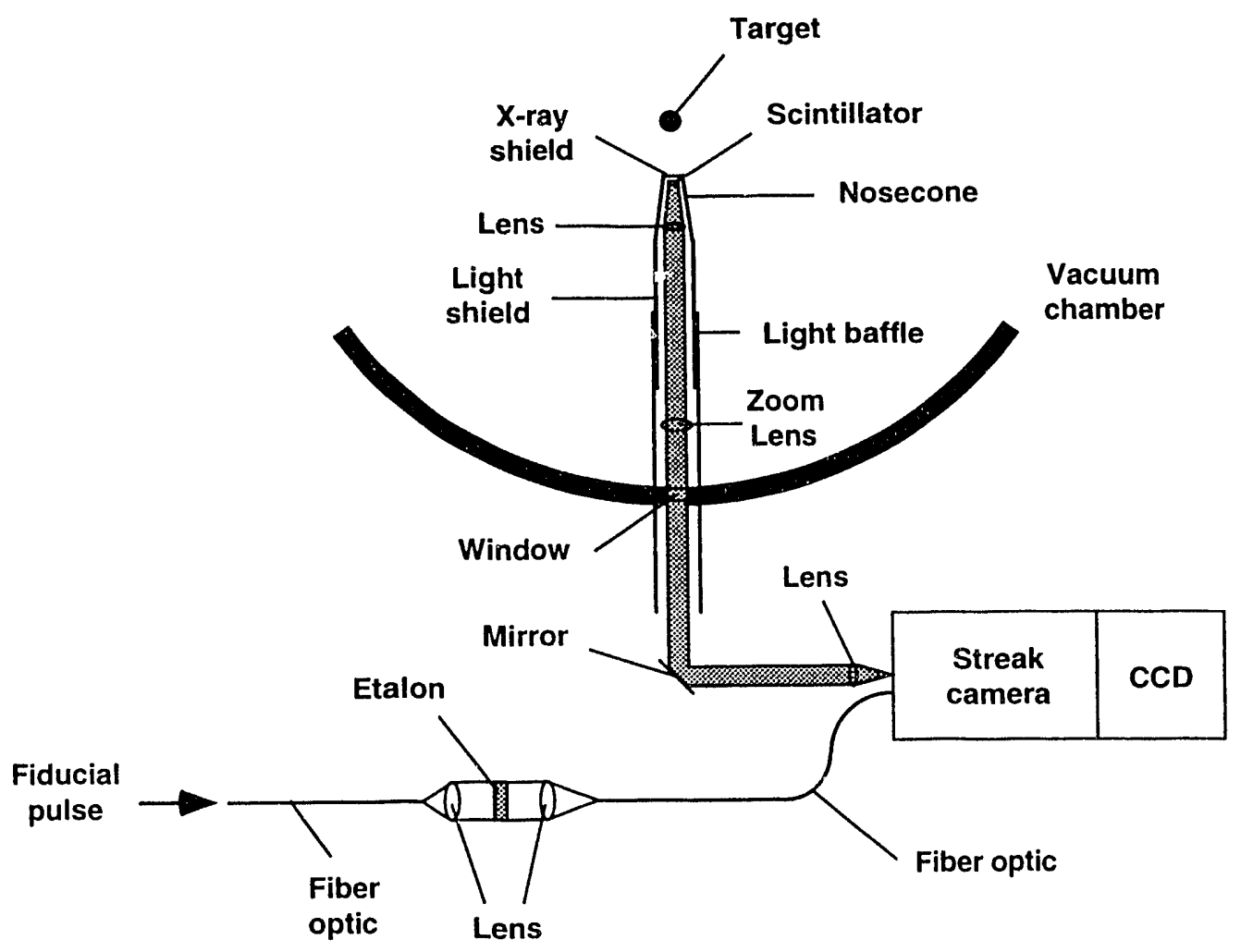

Figure 2. Neutron detector system. Scintillator converts neutron kinetic energy to light. Lens system relays light to a high-speed streak camera for recording.

instruments via fiber optic cables. We insert a 527-ps etalon into the fiducial path to form a series of evenly spaced pulses. The first pulse in the series gives us the required timing reference. It allows us to accurately relate the neutron-signal time base to the laser power history recorded with other streak cameras. We can relate streak camera time bases with about \pm 10 ps accuracy." The pulse train gives us a shot-by-shot check on the stability of the time base. The amplitude and timing of the optical fiducial are easy to control because they are independent of the target type, incident laser energy, and target-to-detector distance. This is not the case when $\mathrm{x}$-rays generated by the target are used as the fiducial.

\subsection{Sensitivity}

Detector sensitivity depends on several factors: the number of neutrons interacting in the useful scintillator volume, the average number of photons generated per interaction, and the efficiency for converting a scintillator photon to a streak tube photoelectron (pe'). In BC-422, about 21,000 photons are generated per detected DT neutron. The light output rate, which is initially about 16 photons per picosecond per detected neutron, decays exponentially with a 1.2-ns time constant. In our system, about $0.058 \mathrm{pe} / \mathrm{ps}$ are generated at the photocathode for each neutron interacting with the scintillator. For this estimate we used the following efficiencies: $1.5 \%$ for the collection efficiency of an $\mathrm{f} / 2$ optic, a signal enhancement of 1.5 for the Al mirror, an optical transmission of $80 \%$, and a photocathode efficiency of $20 \%$. To achieve 20 -ps resolution, signal bin size must be $\leq 10 \mathrm{ps}$. Thus, the system produces about $0.5 \mathrm{pe}^{-}$per resolution element per interacting neutron.

To produce an adequate burn history requires between 100 and 1000 events. To produce 1000 pe per resolution element requires about 2000 neutron interactions. For the useful $1-\mathrm{mm} \times 1.5-\mathrm{mm} \times 6-\mathrm{mm}$ scintillator volume placed $2 \mathrm{~cm}$ from a target, 2000 interactions requires a source strength of about $2 \times 10^{8}$ DT neutrons (neutron mean free path in plastic $\lambda=10 \mathrm{~cm}$ ). Because DD neutrons produce on average only about 1,600 photons per interaction and have half the mean free path, about $7 \times 10^{8} \mathrm{DD}$ neutrons are required for a similar quality signal. 


\subsection{Neutron signals}

Excellent neutron data have been recorded with the instrument. Figure 3 a shows a sample image containing a neutron signal. It was recorded with the scintillator $2 \mathrm{~cm}$ from a target producing $6 \times 10^{\times}$DT neutrons. The fiducial pulse train appears across the top of the image, while the $6-\mathrm{mm}$ wide neutron signal appears directly below. Figure $3 \mathrm{~b}$ shows the image exposure versus time averaged across the spatial extent of the neutron signal. Streak camera flat-field ${ }^{12}$ and time-base ${ }^{13}$ corrections are included in the signal processing. Information about the target burn history is encoded in the leading edge of the pulse; the pulse tail shows the characteristic decay of the scintillator.

The actual neutron temporal distribution at the detector is obtained by deconvolving the effect of the scintillator decaly from the recorded neutron signal. The result of the deconvolution is shown in figure 4a. This fast pulse is approximately the derivative of recorded signal when the temporal width of the radiation exciting the scintillator is much less than the scintillator decaty rate. This deconvolved pulse actually represents the convolution of the neutron temporal distribution with the scintillator rise time and the streak camera response. Since rise time and camera response are both $<20$ ps, the fast pulse is a good representation of the fusion reaction rate. The quality of the deconvolution is checked by convolving the fast pulse (figure 4a) with the exponential decay and comparing the result with the original lineout (figure $3 \mathrm{~b}$ ). This is done in figure $4 b$.

The complete reaction-rate history is plotted in figure 5. The target neutron yield from an independent measurement is used to determine the magnitude of the reaction rate. The fiducial pulse is used to temporally relate the reaction-rate history to the incident laser power used to implode the target. Absolute timing is done with a precision of \pm 10 ps. In this example, the laser drive was nominally a 2 -ns square pulse illuminating a 36()$-\mu m$ diameter glass ball filled with DT gas. The burn history shows the target reached peak emission about $9(0) \mathrm{ps}$ into the laser pulse and that the FWHM of the burn was 120 ps. Also, the burn history is asymmetric. Ior this particular example, the mismatch between laser pulse and target is evident. The last half of the drive pulse arrived at the target after peak compression and burn.
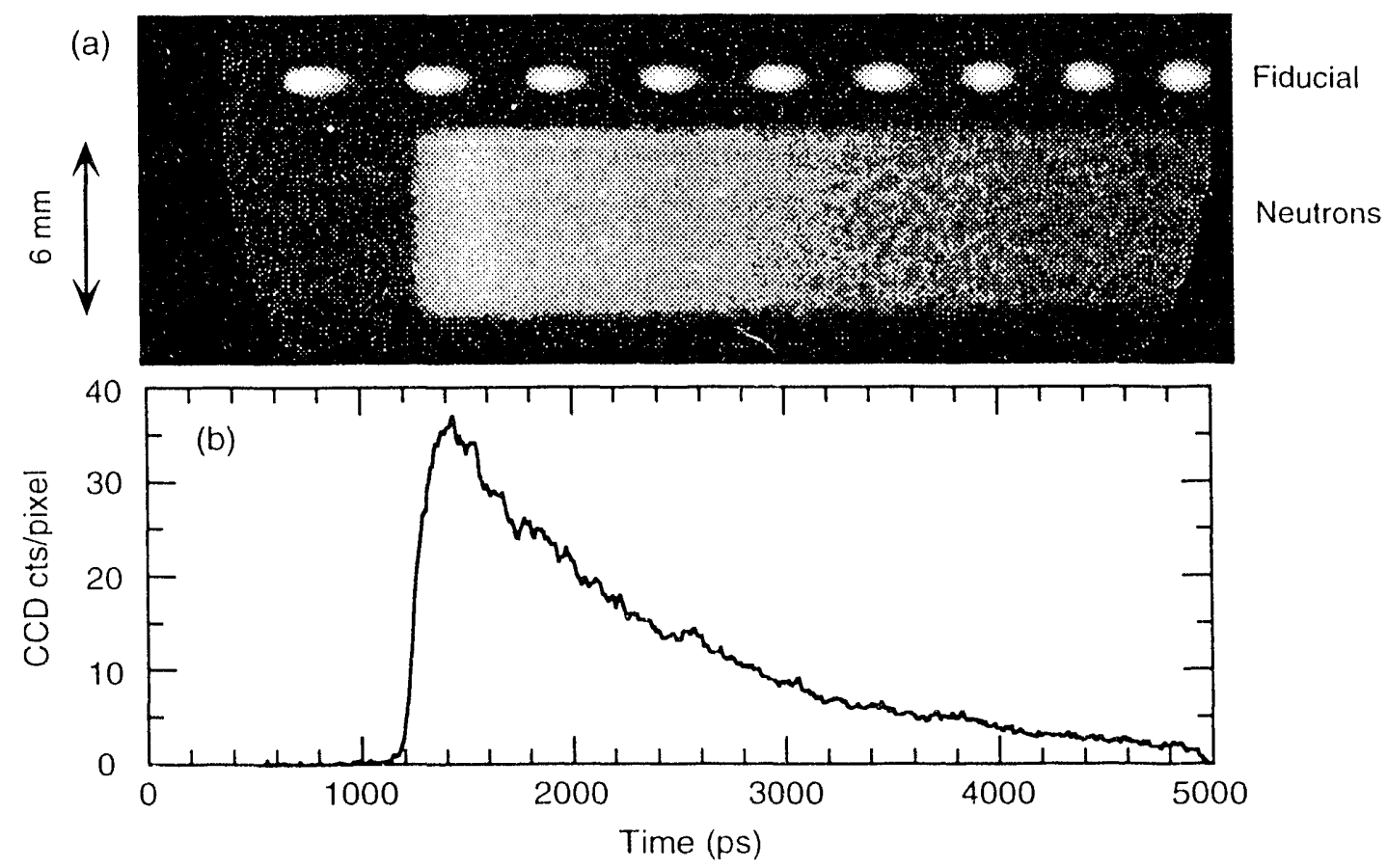

Figure 3. Neutron signal. (a) Streak camera image recorded for target producing $6 \times 10^{\times}$DT neutrons. (b) A verage neutronsignal intensity versus time. Burn history information is encoded in the leading edge of the pulse. The pulse tail shows the characteristic decay of the plastic scintillator. 

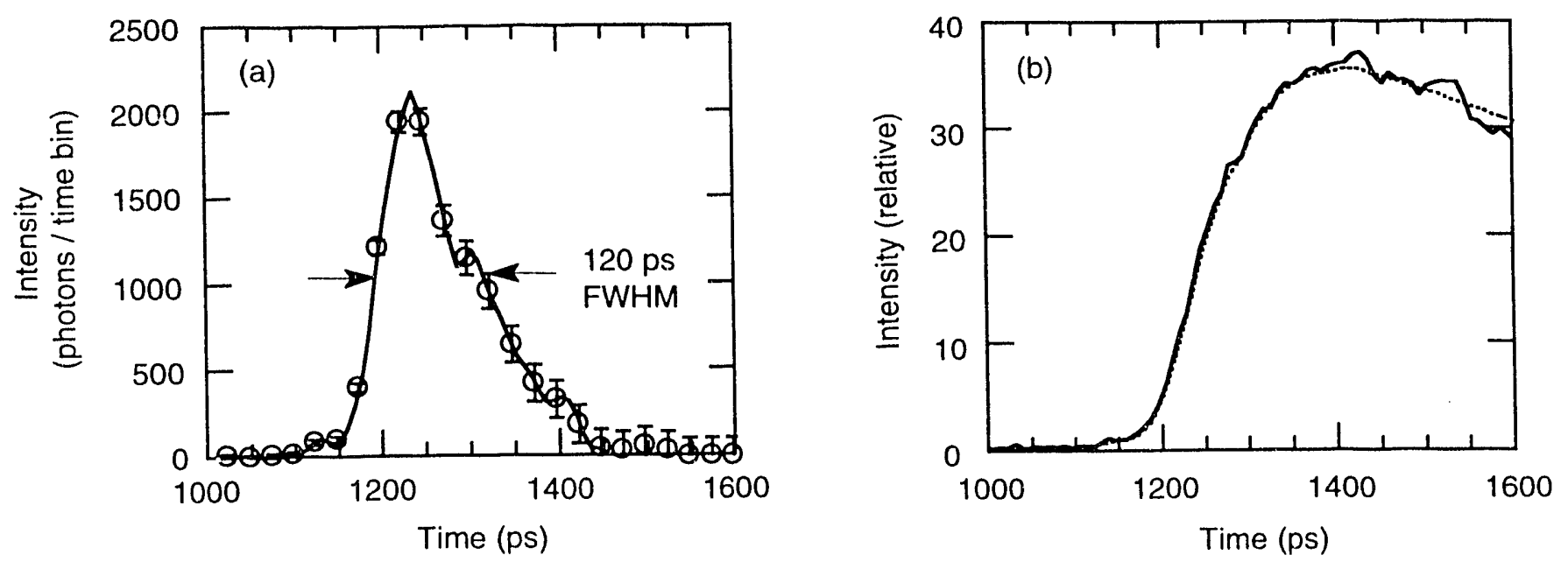

Figure 4. (a) Neutron temporal distribution obtained by deconvolving the exponential decay of the scintillator from the neutron signal shown in figure 3b. (b) Deconvolution check. Fast signal in (a) convolved with the exponential decay (dashed line) compared with original data from figure $3 b$ (solid line).

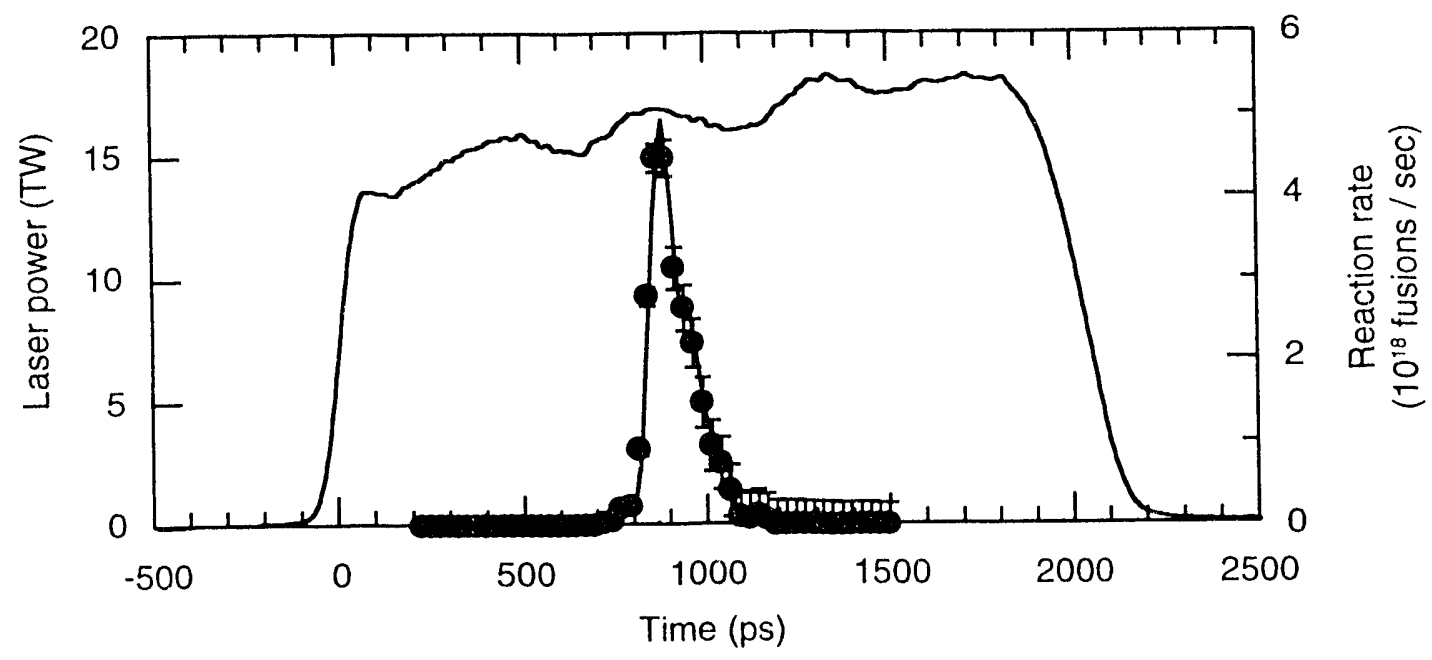

Figure 5. Fusion reaction rate of target relative to the incident laser power at the target.

Detector sensitivity to DT neutrons agrees with the estimate given in section 2.3 . We have recorded usable neutron signals with a reduced temporal resolution of $40 \mathrm{ps}$ for DT neutron yields as low as $3 \times 10^{7}$. Signals have also been recorded for DD-filled targets. These indicate DD-neutron sensitivity is about as expected. We have recorded signals for targets producing as few as $4 \times 10^{\times} \mathrm{DD}$ neutrons. For these DD measurements, the burn history resolution was limited to about $75 \mathrm{ps}$, the neutron transit time through the $1.5-\mathrm{mm}$ thick piece of scintillator.

\subsection{X-ray signal}

The detector response to a short, 20-ps burst of $\mathrm{x}$ rays demonstrates the speed of the scintillator. For this example, the tungsten shield in front of the scintillator was replaced with a thin Be window. A gold disk target was irradiated with a 20-ps laser pulse containing about $70 \mathrm{~J}$ of light at a wavelength of $0.53 \mu \mathrm{m}$. Such targets produces gold $\mathrm{M}$-band $\mathrm{x}$ rays $(2.5-4 \mathrm{keV})$ that pass through the 5-and 10- mil steps of the Be filter and are absorbed in the first micron of the plastic scintillator. The 
(a)

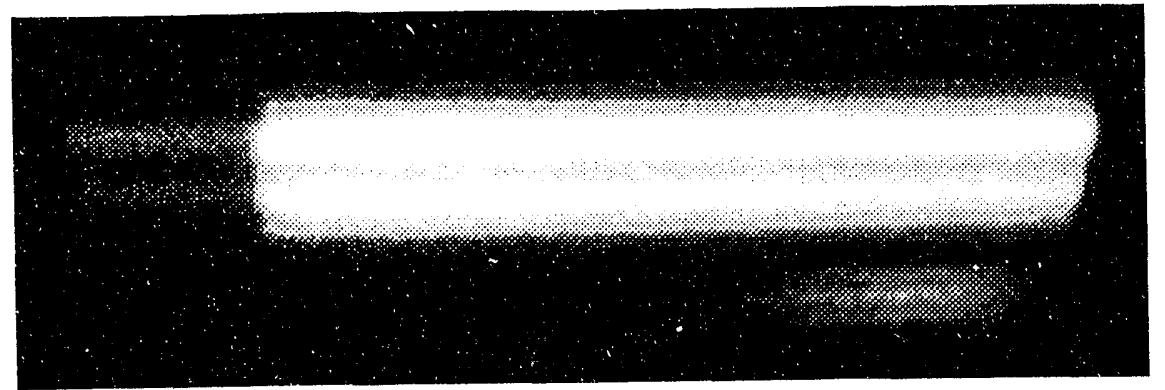

$X$ rays

$0.005^{\prime \prime} \mathrm{Be}$

$0.010^{\prime \prime} \mathrm{Be}$

Fiducial

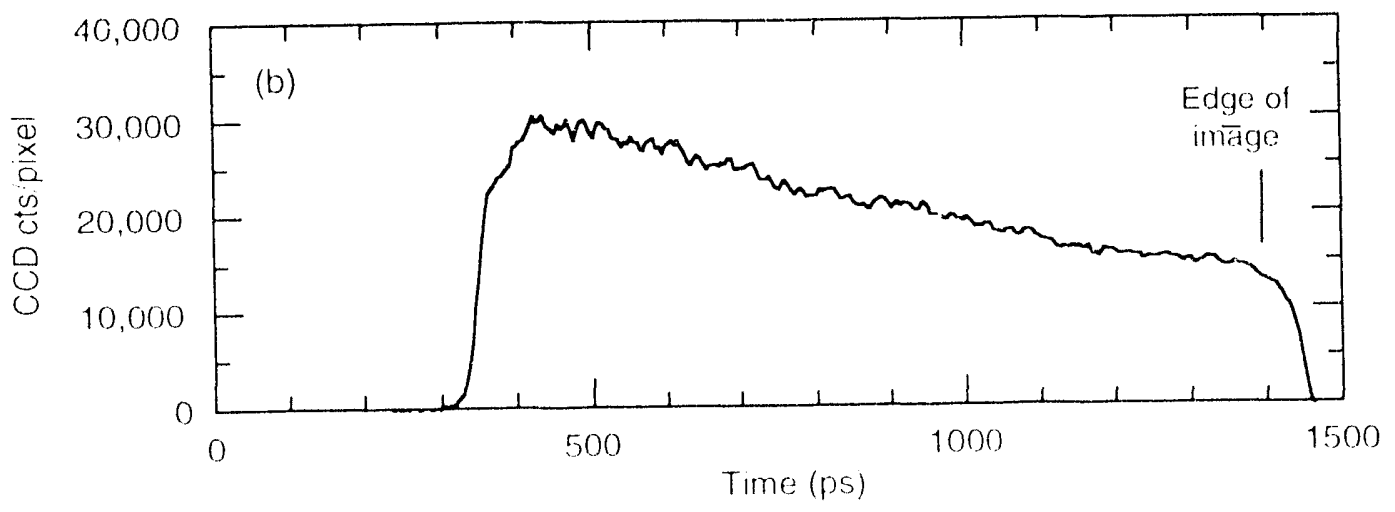

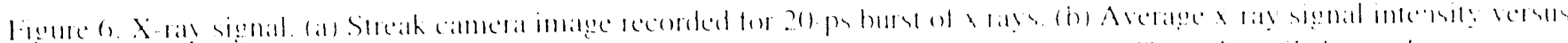

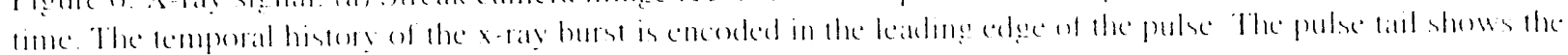

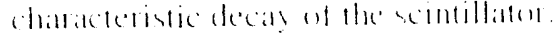
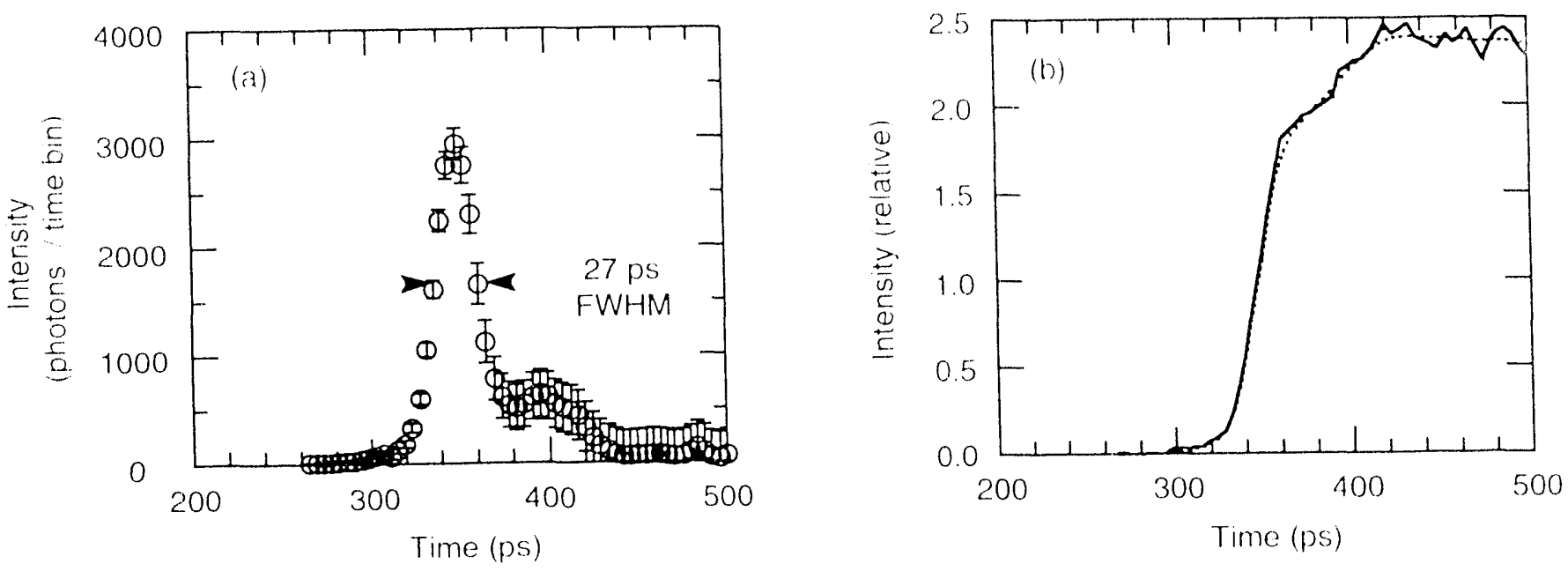

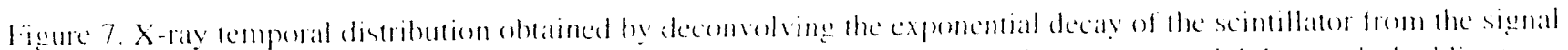
shown in figure ob. (h) Deconvolution check. Fast signal in (a) convolved with the exponemtid decily (dashed line) compatred with origninal data from tigene oh (solid lince). 
recorded image and the $x$-ray intensity versus time are shown in figure 6 . As with the neutron signal, the leading edge of the pulse contains coded information about the temporal distribution of the $x$ rays reaching the scintillator, and the decay of the pulse is characteristic of the scintillator decay.

An unfold of the $x$-ray signal in figure $6 \mathrm{~b}$ indicates the rise time of the plastic scintillator is $<20$ ps. Deconvolution of the exponential decay produces the fast 27-ps FWHM pulse shown in figure 6a. This width represents the convolution of the 20 -ps $x$-ray excitation pulse with a 10 -ps streak camera response and the scintillator rise time. Subtracting the $x$ ray pulse width and streak camera response in quadrature from the 27 -ps pulse gives a pulse width < 16 ps. The integral of a Gaussian pulse has a rise time 1.09 times its FWHM. Thus, the rise time of the plastic scintillator appears to be $<20 \mathrm{ps}$. Also, subtracting in quadrature, the 20-ps drive pulse from the measured 27-ps pulse width indicates overall system response to $x$-rays is $<20$ ps FWHM.

\section{SUMMARY}

We have developed and demonstrated a fast, sensitive neutron detector for measuring the burn history of ICF targets. It is based on the fast rise time of a plastic scintillator and a high-speed streak camera. Its neutron-to-light converter can be conveniently positioned within centimeters of a target. We have demonstrated its temporal resolution to be $<20$ ps FWHM by recording signals generated by 20 -ps bursts of $x$ rays. The detector can measure fusion reactions rates with a resolution of about $25 \mathrm{ps}$ for D'T-filled targets producing as-few-as $10^{\star}$ neutrons. An optical fiducial pulse allows the time base of the neutron signal to be related to the incident laser power with a precision of $\pm 10 \mathrm{ps}$. This detector represents a significant step forward in our ability to measure the reaction-rate histo y for ICF targets.

\section{ACKNOWLIDDGMENTS}

Many people contributed to the successful design and fabrication of the fast neutron detector described in this paper. We especially want to acknowledge R. Ellis for the design of the $f / 2$ achromatic zoom lens system and the alignment system, J. Waldrep and J. Wass for the mechanical layout and design of the instrument, G. Mant for the machining parts and assembling the instrument and its mounting platform, R. Griffith, J. Hatch, and J. Prior for assembly and characterization of the optical streak camera, N. Selchow for specifying and programming the nosecone and zoom lens positioning controls, and D. Kumpt for coordinating purchases of the hardware. We also want to thank M. Cable and T. Murphy for useful discussions and their help with acquiring experimental data. And finally, we want to acknowledge the excellent support we received from the Nova Operations staff, and especially the Laser and Target Diagnostic Techs. This work was performed under the auspices of the U. S. Department of Energy by the Lawrence Livermore National Laboratory under contract number W-7405-ENG-48.

\section{REFERENCES}

I. E. M. Campbell, J. T. Hunt, E. S. Bliss, D. R. Speck, and R. P. Drake, "Nova experimental facility," Re'v. Sci. Instrum. $57(8)$, pp. 2101-2106 (1986).

2. John D. Lindl, Robert L. McCrory, and E. Michael Campbell, "Progress towards ignition and burn piopagation in inertial confinement fusion," Phys. Today 45, 32 (1992).

3. H. Brysk, "Fusion neutron energies and spectra," Plasma Phys. 9, pp. 611-617 (1973).

4. R. A. Lerche, D. R. Kania, S. M. Lane, G. L. Tietbohl, C. K. Bennett, and G. P. Baltzer, "Neutron emission time measurements for ICF targets," Rev. Sci. Instrum. 59 (8), pp. 1697-1699 (1988).

5. D. R. Kania, S. M. Lane, and S. G. Prussin, "Measurements of single 14-MeV neutron bursts with 100 -ps time resolution," Appl. Phy's. Lett. 53, pp. 1988-1989 (1988).

6. H. Azechi, N. Miyanaga, R. O. Stapf, H. Takabe, A. Nishiguchi, M. Unemoto, Y. Shimada, M. Yamanaka, T. Yamanaka, S. Nakai, and C. Yamanaka, "Thermonuclear burn time and duration in laser-driven high-aspect-ratio targets," Appl. Phys. Lett. 55 (10), pp. 945-947 (1989).

7. R. A. Lerche, D. Ress, R. J. Ellis, S. M. Lane, and K. A. Nugent, "Neutron penumbral imaging of laser-fusion targets," Laser and Particle Beams 9 (1), pp. 99-118 (1991).

8. Bicron Corp., Newbury, $\mathrm{OH}$

9. R. A. Lerche and R. L. Griffith, "Resolution limitations and optimization of the LLNL streak camera focus," Procecdings of SPIE High Speed Photography, Videography, and Photonics V, Vol. 832, pp. 266-274 (1987). 
10. B. A. Jones, "A lens coupled streak camera readout system utilizing a thermoelectrically cooled CCD," Proceeding of SPIE High Speed Photography, Videography, and Photonics III, Vol. 569, pp. 189-193 (1985).

11. R. A. Lerche, "Timing between streak cameras with a precision of $10 \mathrm{ps,"} \mathrm{Proceedings} \mathrm{of} \mathrm{SPIE} \mathrm{High-Speed}$ Photography, Videography, ar,d Photonics and Velocimetry '90, Vol. 1346, pp. 376-383 (1990).

12. J. A. Hatch, D. S. Montgomery, and J. Prior, "Long term flat-field behavior in LLNL streak camers: preliminary results," Proceedings of SPIE High-Speed Photography, Videography, and Photonics and Velocimetry "90, Vol. 1346, pp. 371-375 (1990).

13. D. S. Montgomery, R. P. Drake, R. A. Lerche, C. W. Laumann, and J. D. Wiedwald, "Evaluating the accuracy of optical-streak-camera sweep rates using uncertain data," Rev. Sci. Instrum. 63 (10), pp. 4322-4326 (1992). 

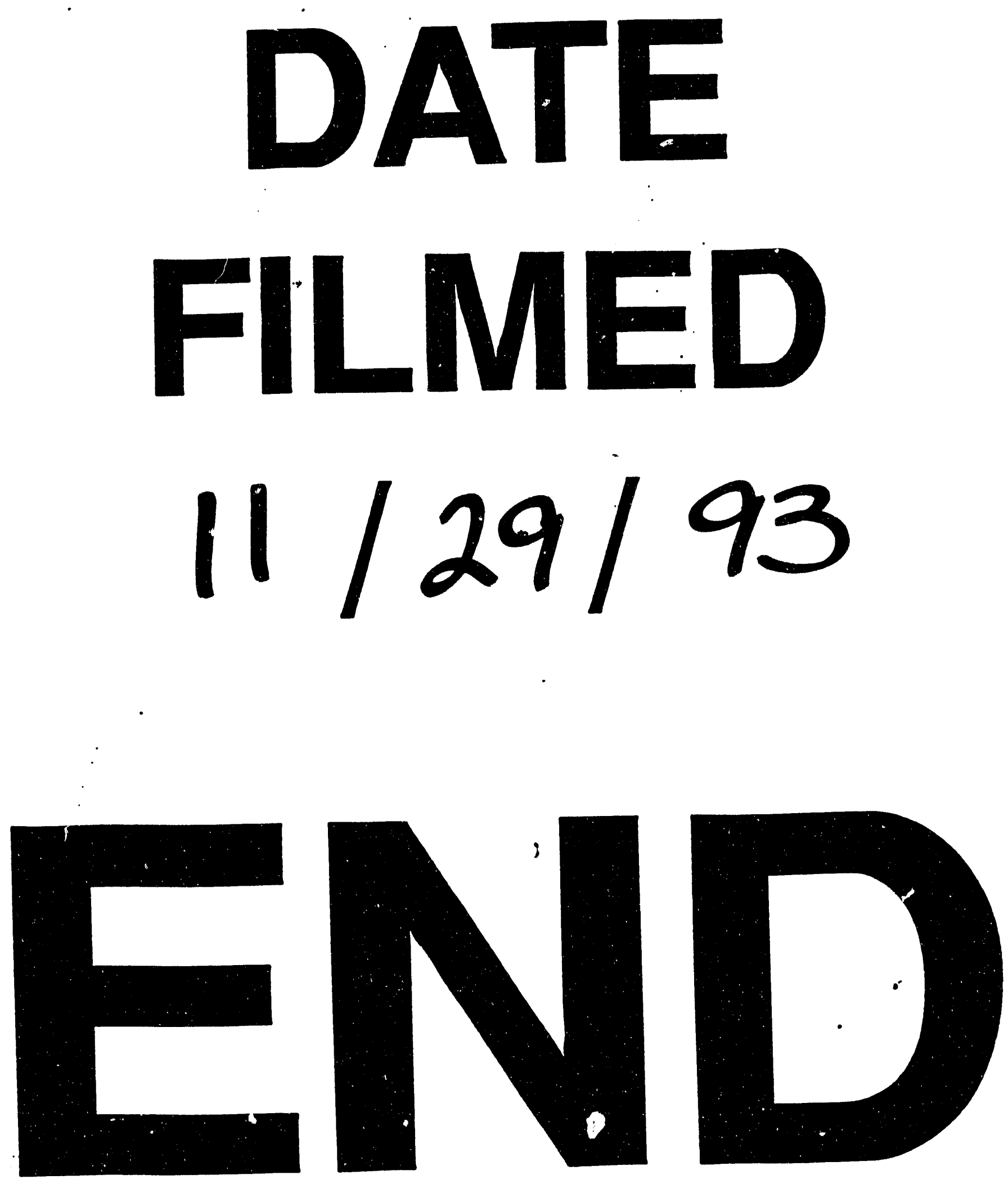
